\title{
Comparison of the combustion behaviors of agricultural wastes under dry air and oxygen
}

\author{
Hanzade Haykiri-Acma ${ }^{1, *}$, Serdar Yaman ${ }^{1}$ \\ ${ }^{1}$ Department of Chemical Engineering, Istanbul Technical University, Istanbul, Turkey \\ *Corresponding author. Tel: +90 2122856291, Fax:+90 2122852925, E-mail:hanzade@itu.edu.tr
}

\begin{abstract}
Burning tests of some agricultural waste biomass materials such as sunflower seed shell (SSS), hazelnut shell (HS), rice hull (RH), and olive refuse (OR) were performed in order to compare the combustion reactivities of these materials under dry air and oxygen. For this purpose, these samples were burned in a thermal analyzer to obtain TGA (Thermogravimetric Analysis), DTG (Derivative Thermogravimetry), DTA (Differential Thermal Analysis), and DSC (Differential Scanning Calorimetry) thermograms under both conditions. Initial sample mass was approximately $10 \mathrm{mg}$ for each sample which has a particle size of $<0.25 \mathrm{~mm}$, and temperature was raised from ambient to $900^{\circ} \mathrm{C}$ with a linear heating rate of $40^{\circ} \mathrm{C} / \mathrm{min}$ under gas flow rate of $100 \mathrm{~mL} / \mathrm{min}$. No hold time was allowed at the final temperature. The results of this study showed that the thermal reactivities of biomass species change in a wide region, and the type of the oxidative medium plays very important effects on the burning parameters such as the ignition point, maximum rate of combustion and its temperature, as well as the end point of burning. On the other hand, macromolecular ingredients of biomass such as hemicellulosics, celluloses, and lignin have significant effects on the combustion behavior of biomass.
\end{abstract}

Keywords: Biomass, Agricultural Wastes, Combustion, Dry Air, Oxygen

\section{Introduction}

There are several methods to control $\mathrm{CO}_{2}$ emissions emitted from power plants including precombustion capture, post-combustion capture, and oxy-fuel combustion. Pre-combustion capture is also called as gasification or partial reforming for which fuel is reacted with air, oxygen, or steam to produce gaseous products. Post-combustion techniques are based on scrubbing of $\mathrm{CO}_{2}$ from the flue gas. In oxy-fuel or $\mathrm{O}_{2} / \mathrm{CO}_{2}$ recycle combustion method, nitrogen is excluded using pure $\mathrm{O}_{2}$ instead of air [1,2]. In oxy-fuel technique, the diluting effect of nitrogen in the flue gas does not take place, and the concentration of $\mathrm{CO}_{2}$ becomes likely high which is suitable for $\mathrm{CO}_{2}$ capture and storage (CCS) techniques [3]. That is, the concentration of $\mathrm{CO}_{2}$ in the flue gas from conventional coal-fired boilers typically changes in the range of 4-14 vol \% [1], whereas in case of oxy-fuel its concentration increases to 55-65 vol \%. Then, cooling and condensing of water vapor leads to increase in the $\mathrm{CO}_{2}$ concentration to around $96 \mathrm{vol} \%$ [4].

On the other hand, high concentrations of $\mathrm{CO}_{2}$ in the combustion medium alter the combustion properties compared to $\mathrm{N}_{2}$-rich combustion medium. Ignition temperature, burner stability, flame propagation, gas temperature, the char burnout, the radiating properties of the flame, the efficiency of the boiler, and the evolution of pollutants are closely affected from the high concentration of $\mathrm{CO}_{2}$ [5]. Some of these alterations are directly associated with the increasing radiative properties and the thermal capacity of the mixture of $\mathrm{CO}_{2}$ and water vapor compared to $\mathrm{N}_{2}$. Combustion kinetics also changes considerably under high $\mathrm{CO}_{2}$ concentration [6]. From this point of view, combustion characteristics of a given fuel species should be evaluated depending on the type of oxidizer.

So the aim of this paper is to investigate the differences in the combustion properties of some agricultural wastes using dry air or pure oxygen. For this purpose, sunflower seed shell, hazelnut shells, rice husks, and olive refuse have been selected as the model biomass species, 
all of which are abundant in Turkey and they have already been used for energy resources for a long time.

\section{Methodology}

Agricultural biomass energy resources such as sunflower seed shell (SSS), hazelnut shells (HS), rice husks $(\mathrm{RH})$, and olive refuse from milling (OR) used in this study are Turkish origin. These renewable energy sources were not dried in oven to avoid any modification in their original structure due to rapid drying, and they were kept at laboratory medium for 15 days to allow removal of the free moisture. Then, air-dried samples were milled and screened through a sieve having an opening of $250 \mu \mathrm{m}$. The proximate analysis and the gross calorific value measurements of the biomass species were carried out according to ASTM standards, and the ultimate analyses were performed by an elemental analyzer (EuroEA3000 model). These tests were repeated several times to check the reproducibility of the results.

The main ingredients of biomasses such as holocellulose (hemicellulosics + cellulose forms), lignin, and extractive matter were determined by analytical methods according to the following procedures. In order to remove the extractives and to obtain extractives-free samples, benzene-ethyl alcohol extraction procedure was applied according to ASTM D1105 standard.

The extractives-free bulk was then used as feedstock to isolate each of holocellulose and lignin. Isolation of holocellulose was performed with the mixtures of $\mathrm{NaClO}_{2}$, acetic acid, and water. Whereas, the isolation of lignin was carried out by van Soest method in which extractives-free sample was treated with $72 \mathrm{vol} \%$ sulphuric acid to hydrolyze the cellulosics and to isolate the lignin [7]. The content of acid insoluble lignin which is called as "Klason Lignin” was determined by drying and ashing of the neutralized bulk.

Combustion tests of the samples were performed using a TA Instruments SDTQ600 model thermogravimetric analyzer with a differential scanning calorimetry detector. TGA (Thermogravimetric Analysis), DTG (Derivative Thermogravimetry), DTA (Differential Thermal Analysis), and DSC (Differential Scanning Calorimetry) thermograms were obtained using dry air or oxygen at flow rates of $100 \mathrm{~mL} / \mathrm{min}$, and the initial weights of the samples were around $10 \mathrm{mg}$. Temperature was increased from ambient to $900^{\circ} \mathrm{C}$ by a heating rate of $40^{\circ} \mathrm{C} / \mathrm{min}$, and no hold time was allowed.

\section{Results and Discussion}

Analysis results of the samples are seen in Table 1. According to data given in Table 1, it can be said that all the biomass species are rich in volatiles and their fixed carbon contents are considerably lower than the contents of volatiles. In fact, such a distribution of the contents of volatile matter and fixed carbon is typical for most biomass species [8]. SSS is the biomass material that contains the highest volatiles among the samples. Ash contents of the samples varies in a so wide range that the ash content of SSS is only $2.7 \%$ while the ash content of $\mathrm{RH}$ reaches $23.8 \%$. Sulfur contents of the biomass species are very low regarding the ash contents of the low rank coals in general. On the other hand, hydrogen and nitrogen contents of all the samples are very close to each other.

Lignin contents of the biomass materials are also very close to each other except for HS. Although, the lignin contents of SSS, RH, and OR changes between 31.4 and $34.8 \%$, HS which has a woody structure contains higher lignin content as much as $51.5 \%$. Besides, SSS 
which gives the highest volatiles yield also contains the highest holocellulose content. In addition, the lowest calorific value belongs to $\mathrm{RH}$ that is rich in ash forming mineral matter.

Table 1. Analysis results of the biomass species

\begin{tabular}{|c|c|c|c|c|c|}
\hline \multirow{4}{*}{$\begin{array}{l}\text { Proximate Analysis } \\
\text { (\%, dry basis) }\end{array}$} & & SSS & HS & $\mathrm{RH}$ & OR \\
\hline & Volatiles & 83.7 & 72.0 & 66.2 & 71.2 \\
\hline & Fixed Carbon & 13.6 & 21.0 & 10.0 & 14.6 \\
\hline & Ash & 2.7 & 7.0 & 23.8 & 14.2 \\
\hline \multirow{5}{*}{$\begin{array}{l}\text { Ultimate Analysis } \\
\text { (\%, dry-ash-free basis) }\end{array}$} & $\mathrm{C}$ & 47.8 & 54.8 & 44.8 & 49.3 \\
\hline & $\mathrm{H}$ & 6.1 & 6.7 & 6.3 & 6.2 \\
\hline & $\mathrm{N}$ & 1.2 & 1.0 & 0.9 & 1.7 \\
\hline & $\mathrm{S}$ & 0.3 & 0.1 & 0.1 & 0.1 \\
\hline & $\mathrm{O}^{*}$ & 44.6 & 37.4 & 47.9 & 42.7 \\
\hline \multirow{3}{*}{$\begin{array}{l}\text { Structural Analysis } \\
\text { (\%, dry basis) }\end{array}$} & Extractives & 13.8 & 6.2 & 9.8 & 13.6 \\
\hline & Lignin & 31.4 & 51.5 & 34.8 & 34.7 \\
\hline & Holocellulose & 62.5 & 38.6 & 44.9 & 40.0 \\
\hline Calorific Analysis & $\begin{array}{l}\text { Higher Calorific } \\
\text { Value }(\mathrm{MJ} / \mathrm{kg})\end{array}$ & 17.7 & 18.2 & 13.9 & 17.2 \\
\hline
\end{tabular}

* calculated by difference

DTG and DSC curves obtained from non-isothermal thermal analyses of the biomass samples under dry air are illustrated in Fig.1.
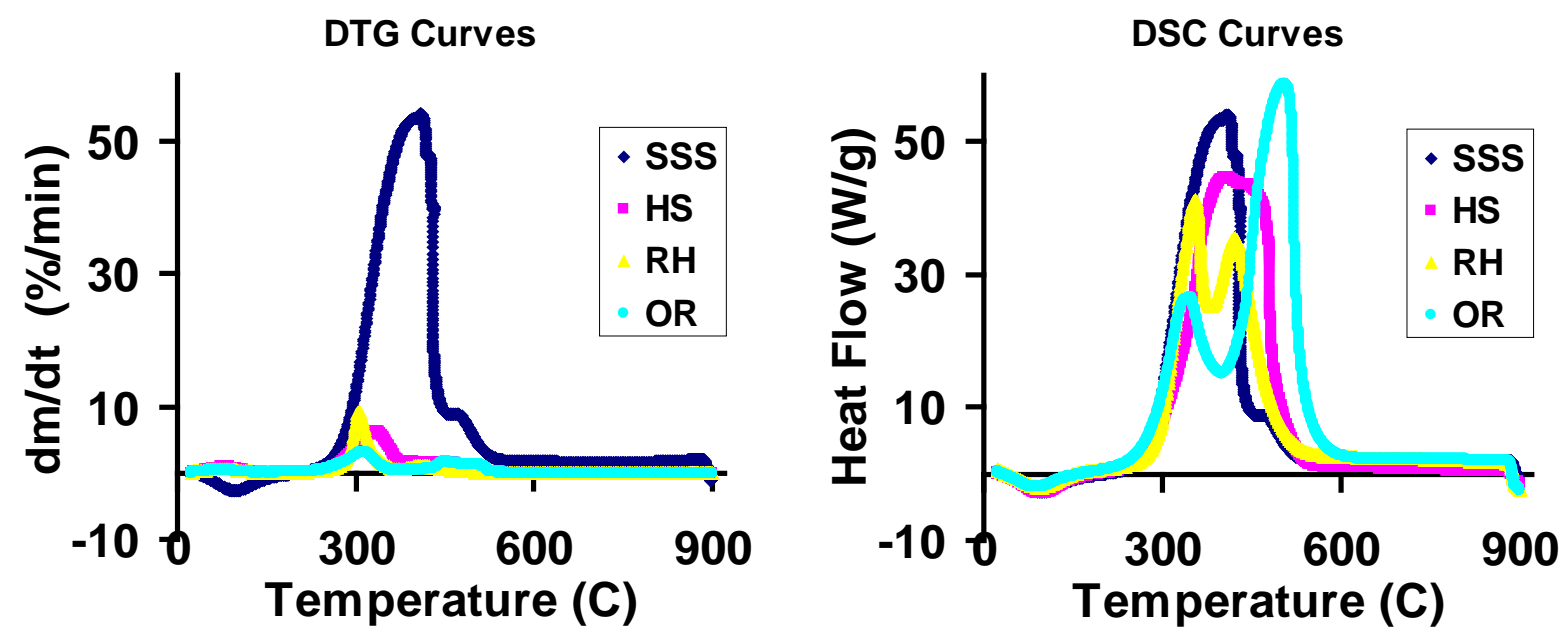

Fig. 1. DTG and DSC curves obtained from burning with dry air.

DTG curves which are seen on the left hand side of Fig.1 show the relation between temperature and the rates of the mass losses from the biomass samples. These curves indicated that the thermal decomposition and the burning of SSS have such a characteristics that it losses the weight so rapidly that its maximum rate of burning reaches $53.6 \% / \mathrm{min}$ at 
$413^{\circ} \mathrm{C}$. Besides, the maximum burning rates for the other samples could not be at this level that they were $8.2 \% / \mathrm{min}$ at $320^{\circ} \mathrm{C}$ for $\mathrm{RH}, 6.3 \% / \mathrm{min}$ at $307^{\circ} \mathrm{C}$ for $\mathrm{HS}$, and $3.2 \% / \mathrm{min}$ at 314 ${ }^{\circ} \mathrm{C}$ for OR. Although the mass losses from the samples continued as temperature increases up to the final temperature, they are negligible beyond $600^{\circ} \mathrm{C}$. The high thermal reactivity and the very high rates of mass losses from SSS can be attributed to the high contents of volatiles in this sample. In fact, high contents of holocellulose which is sum of hemicellulosics and cellulosics contribute to the formation of volatiles [9]. These constituents which are rich in weak ether bonds are thermally unstable and they produce volatile species. Of which, combustible volatiles are able to burn in the gaseous phase as homogeneous combustion. Elimination of the volatiles from the solid matrix leads to the formation of porous remnant and then burning of the char takes place firstly on the surface which is followed by diffusion of oxygen into the pores and complete burning of the particles. The latter is generally called as the heterogeneous burning stage [10]. Thus, all the organic part of the samples could be oxidized until the end of the burning experiment since the final temperature was high enough for combustion of most biomass materials.

On the other hand, the heat flows which are shown as DSC curves on the right hand side of Fig.1 predicts that the huge rates of mass losses in the DTG curve for SSS could not contribute to the exothermic performance of this sample at expected level. This is because the most of the mass losses are formed from the elimination of the volatiles such as carbon dioxide which play no important role on the calorific output. The exothermic regions for all the samples either comprised of two different parts or a unique broad peak having a shoulder, representing the effects of both homogeneous combustion of volatiles and char burning.

In order to investigate the individual effects of biomass ingredients on burning, each of the isolated ingredients including holocellulose, lignin, and extractive-free samples were burned under dry air condition. Fig.2 represents the burning characteristics of the ingredients of sunflower seed shell and rice husk, the burning properties of which were highly different in their parent samples.

The ingredients for both samples showed similar trends below $250^{\circ} \mathrm{C}$ that almost all of the ingredients lost the same weight in this stage. Increasing temperature affected the weight losses in different way that holocellulose and extractives-free sample of SSS rapidly lost weight while higher temperatures necessitated getting the similar decomposition yield for the lignin content of SSS. On the other hand, decompositions of holocellulose and lignin contents for $\mathrm{RH}$ exceeded the decomposition of extractives-free sample from $250^{\circ} \mathrm{C}$ to the end of the experiment. In this context, the high ratio of ash for $\mathrm{RH}$ is effective at this point, since most of the ash forming minerals still exist after treatment with benzene-ethyl alcohol. Accordingly, it is possible to conclude that the burning yields for the ingredients of SSS are higher than those for RH. This shows that the complex structure of biomass which is comprised of mainly from the major macromolecular ingredients are closely affected from the individual behaviors of the each ingredient during thermal process.

DTG and DSC curves obtained from the burning experiments in which oxygen were used instead of dry air are given in Fig.3. 

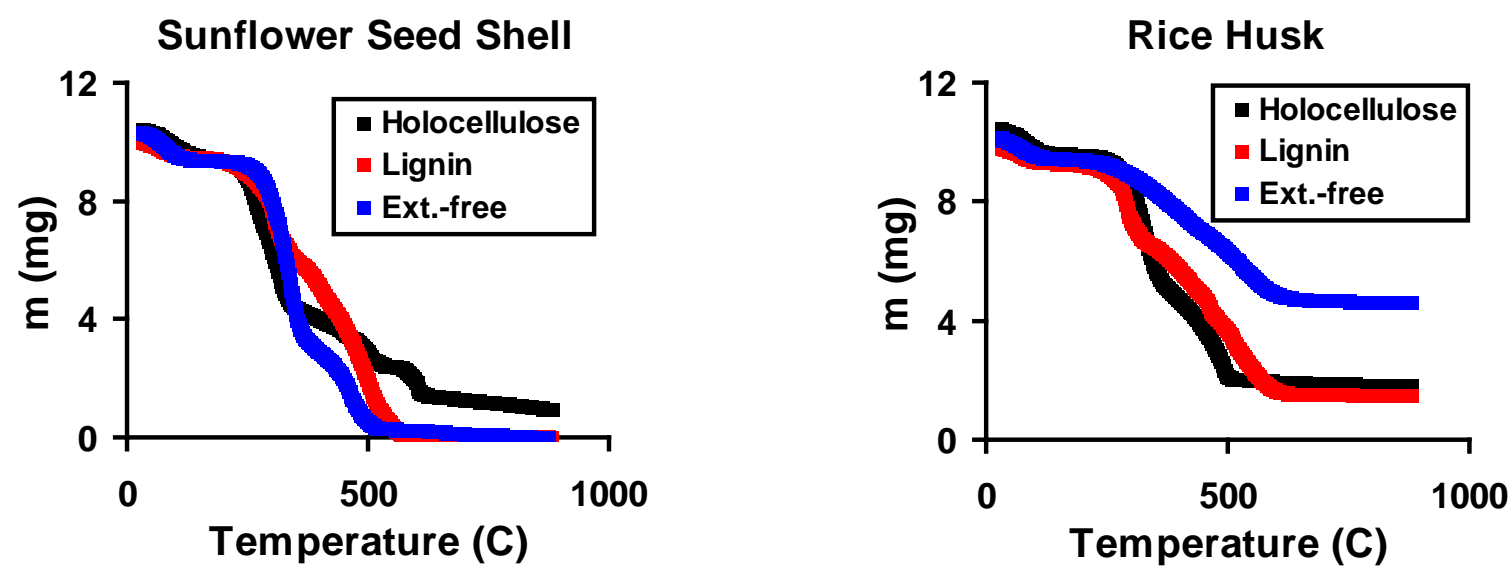

Fig. 2. TGA curves for the Ingredients of SSS and RH under dry air.
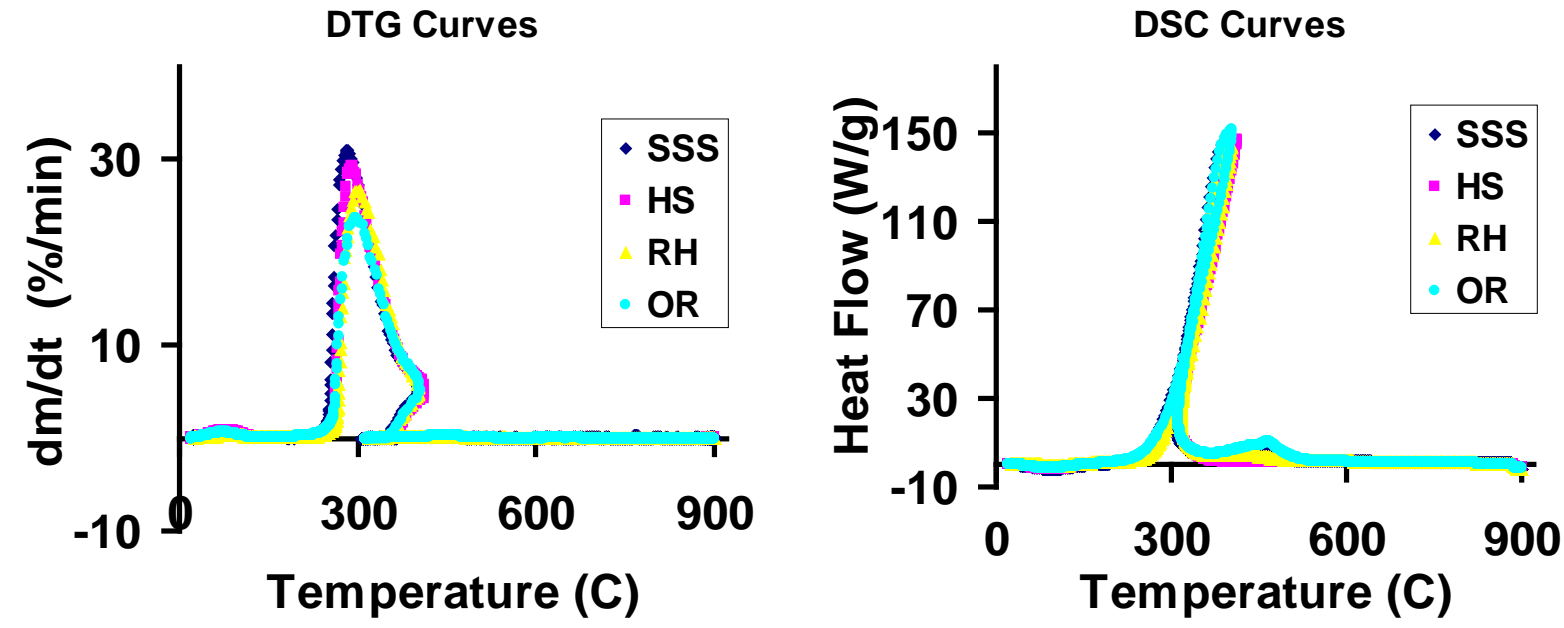

Fig. 3. DTG and DSC curves obtained from burning with oxygen

At the first sight it is likely to conclude that the difference among the DTG curves of the biomass species encountered for burning using dry air wholly disappeared, and the DTG curves almost overlapped in case of oxygen. Also, the burning rates for all the biomass samples except SSS increased more than three-folds when oxidizing gas changed from dry air to oxygen. This shows that usage of pure oxygen during burning of biomass so augmented the thermal reactivity that very different burning profiles could be obtained. Furthermore, the combustion process ended at lower temperatures. These findings can be supported by the results found from the DSC curves. That is, usage of pure oxygen so changed the shapes of the heat flow curves that they almost became very sharp peaks in contrast to the shapes of DSC curves for dry air which had some apparent regions in which heat flows take place. The exothermic heat flows occurred in so narrow temperature intervals that the temperatures of the 
lower and upper limits of these regions are very close to each other. Therefore, it is very difficult to distinguish the individual DSC curves as well as DTG profiles. These results predict that not only the rates of the mass losses but also the heat flows are seriously influenced from the type of the oxidizer medium. Increase in the concentration of oxygen caused variations in thermal behavior of biomass in the favor of increasing reactivity.

\section{Conclusions}

Burning characteristics of some agricultural waste biomass species such as sunflower seed shell, hazelnut shell, rice husk, and olive refuse have been tested under dynamic flows of dry air or oxygen under relatively slow heating conditions in a thermal analyzer. These tests indicated that both the rates of the mass losses from the biomass samples and the heat flow properties are obviously different for each biomass material under dry air. For an example, sunflower seed shell showed such a different weight loss character from the other biomass samples under dry air that it is possible to say that its thermal reactivity is extremely higher than that for the other samples under investigated conditions. Despite this big difference in weight loss characteristics of SSS, heat flow properties determined from DSC curves could not monitored at expected level, and all the samples showed similar heat flow characteristics to some extent. The major ingredients of biomass samples including holocellulose and lignin plays a significant role on the thermal reactivity and the exothermic characteristics of the burning process.

On the other hand, a different situation was detected in the DTG and DSC curves obtained under pure oxygen. That is, almost all the DTG curves for the samples overlapped to form a unique peak as well as the DSC curves. This shows that usage of oxygen instead of dry air eliminated the differences in the thermal reactivity and the burning features of the biomass species under investigated conditions. Also, thermal reactivities of biomasses seriously increased in case of oxygen usage.

\section{References}

[1] J. Davison, K. Thambimuthu, Technologies for capture of carbon dioxide, in: Greenhouse Gas Control Technologies, Vol.I, Elsevier, 2005, pp. 3-13.

[2] R. Allam, V. White, N. Ivens, M. Simmonds, Heaters and boilers to oxyfiring by cryogenic air separation and flue gas recycle, in : Carbon Dioxide Capture for Storage in Deep Geologic Formations, Vol. 1, Elsevier, 2005, pp.451-475.

[3] G. Pipitone, O. Bolland, Power generation with $\mathrm{CO}_{2}$ capture: Technology for $\mathrm{CO}_{2}$ purification, International Journal of Greenhouse Gas Control 3, 2009, pp. 528-534.

[4] I. Hadjipaschalis, G. Kourtis, A. Poullikkas, Assessment of oxyfuel power generation technologies, Renewable and Sustainable Energy Reviews 13, 2009, pp. 2637-2644.

[5] P.A. Bejarano, Y.A. Levendis, Single-coal-particle combustion in $\mathrm{O}_{2} / \mathrm{N}_{2}$ and $\mathrm{O}_{2} / \mathrm{CO}_{2}$ environments, Combustion and Flame 153, 2008, pp. 270-287.

[6] T. Wall, Y. Liu, C. Spero, L. Elliott, S. Khare, R. Rathnam, F. Zeenathal, B. Moghtaderi, B. Buhre, C. Sheng, R. Gupta, T.Yamada, K. Makino, J. Yu, An overview on oxyfuel coal combustion-State of the art research and technology development, Chemical Engineering Research and Design 87, 2009, pp. 1003-1016.

[7] P.J. Van Soest, Use of detergents in the analysis of fibrous feeds. II. A rapid method for the determination of fiber and lignin. Journal of Association of Official Analytical Chemistry. 46, 1963, pp. 829-835. 
[8] D.L. Klass, Biomass for renewable energy, fuels, and chemicals, Academic Press, 1998, pp. 237-239.

[9] R.Kandiyoti, A.Herod, K.Bartle, Solid fuels and heavy hydrocarbon liquids; thermal characterization and analysis, Elsevier, 2006, p.38.

[10]B.G.Miller, D.A. Tillman, Combustion engineering issues for solid fuel systems, Elsevier, 2008, pp.17-21. 